\title{
Characteristics of abnormal large waves measured from coastal videos
}

\author{
J. Yoo ${ }^{1}$, D.-Y. Lee ${ }^{1}$, T.-M. Ha ${ }^{2}$, Y.-S. Cho ${ }^{2}$, and S.-B. Woo $^{3}$ \\ ${ }^{1}$ Korea Ocean Research and Development Institute, Ansan, Korea \\ ${ }^{2}$ Department of Civil \& Environment Engineering, Hanyang University, Seoul, Korea \\ ${ }^{3}$ Department of Oceanography, Inha University, Incheon, Korea
}

Received: 13 January 2010 - Revised: 5 April 2010 - Accepted: 16 April 2010 - Published: 29 April 2010

\begin{abstract}
Recently, tsunami-like sea-level oscillations occurred in a region of the west coast of Korea (i.e. in the eastern part of the Yellow Sea), during a typical rough spring weather episode on 4 May 2008. The analysis of these tsunami-like abnormal waves focuses solely on the videos recorded by a CCTV surveillance system in the directions of the entrance and inside parts of a local coastal pocket beach. Time-series of the vertical and horizontal sea surface oscillations were extracted from the video recordings through calibrating image distortions, accumulating 1-D intensity arrays along the line transects of interest in time, and identifying the trajectories of the oscillations. Frequency and time-domain analysis of the time-series signals revealed that the maximum height of the tsunami-like waves reached $1.3 \mathrm{~m}$, having a dominant period of $185 \mathrm{~s}$ ( $3.1 \mathrm{~min})$. In addition, the results indicate that the celerity of the maximum height wave approximated $7.3 \mathrm{~m} / \mathrm{s}$, which lead to the losses of life of several people who could not escape immediately from the fast tsunami flooding the shore.
\end{abstract}

\section{Introduction}

Extremely large and long waves, commonly known as tsunami, can be generated by a variety of geo-mechanical sources, such as earthquakes, submarine landslides, asteroid impacts, and atmospheric disturbances (Monserrat et al., 2006). As these waves with a typical period range of minutes to hours approach the coast, they may result in severe flooding of coastal residences and destructive damage to coastal structures. It is worth noting that many of the ordinary tsunami events tend to be observed in the vulnerable coasts over a large spatial scale (greater than $10 \mathrm{~km}$ ), or over a transoceanic coverage. In contrast, atmospherically-

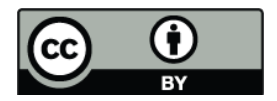

Correspondence to: J. Yoo (jyoo@kordi.re.kr) generated tsunami waves have been typically observed in specific harbors, bays and inlets around the world (Hibiya and Kajiura, 1982; Wang et al., 1987; Rabinovich, 1993; Papadopoulos, 1993; Dragani et al., 2002; Gorging, 2005; among others).

Meteorological tsunami waves are reported to occur as abnormally strong sea level oscillations in those coastal pocket areas where resonant open-ocean incoming waves are coupled with traveling atmospheric disturbance that have frequency bands similar to ordinary tsunami waves (Vilibic and Paklar, 2006). Strong sea level oscillations are increasingly excited by the preferred shape and geometry of a coastal basin. Atmospheric gravity perturbations can be caused by various atmospheric processes: atmospheric gravity waves, pressure jumps, frontal passages, squalls, storms, and, in extreme cases, dynamic instability provoked by wind shear in the lower atmospheric layer (Monserrat and Thorpe, 1992).

Recently, a possible example of meteorological tsunami waves occurred in a local pocket beach of the west coast of Korea (i.e. in the eastern part of the Yellow Sea, as shown in Fig. 1), amid a typical spring weather episode, on 4 May 2008. The abnormal large sea level oscillations, as reported by on-site eyewitnesses, lasted for tens of minutes, and the tsunami-like largest amplitude waves during the abnormal oscillations washed 36 people back into the ocean who were staying near the breakwater constructions along the pocketshaped coast. The locally disastrous wave event was not associated with any earthquake nor considerable storm activity was reported in the Yellow Sea. Considering atmospheric instabilities revealed from the meteorological data collected on the event day (Oh et al., 2008), it is accepted that the large oscillations were induced by the passing of an abrupt air pressure jump over the pocket beach region (Fig. 2a). Previously, other similar cases of abnormally large and long waves were documented as occasional occurrences in regions on the west coast of Korea, in particular, under more or less average rough spring weather conditions (Choi et al., 2008).

Published by Copernicus Publications on behalf of the European Geosciences Union. 

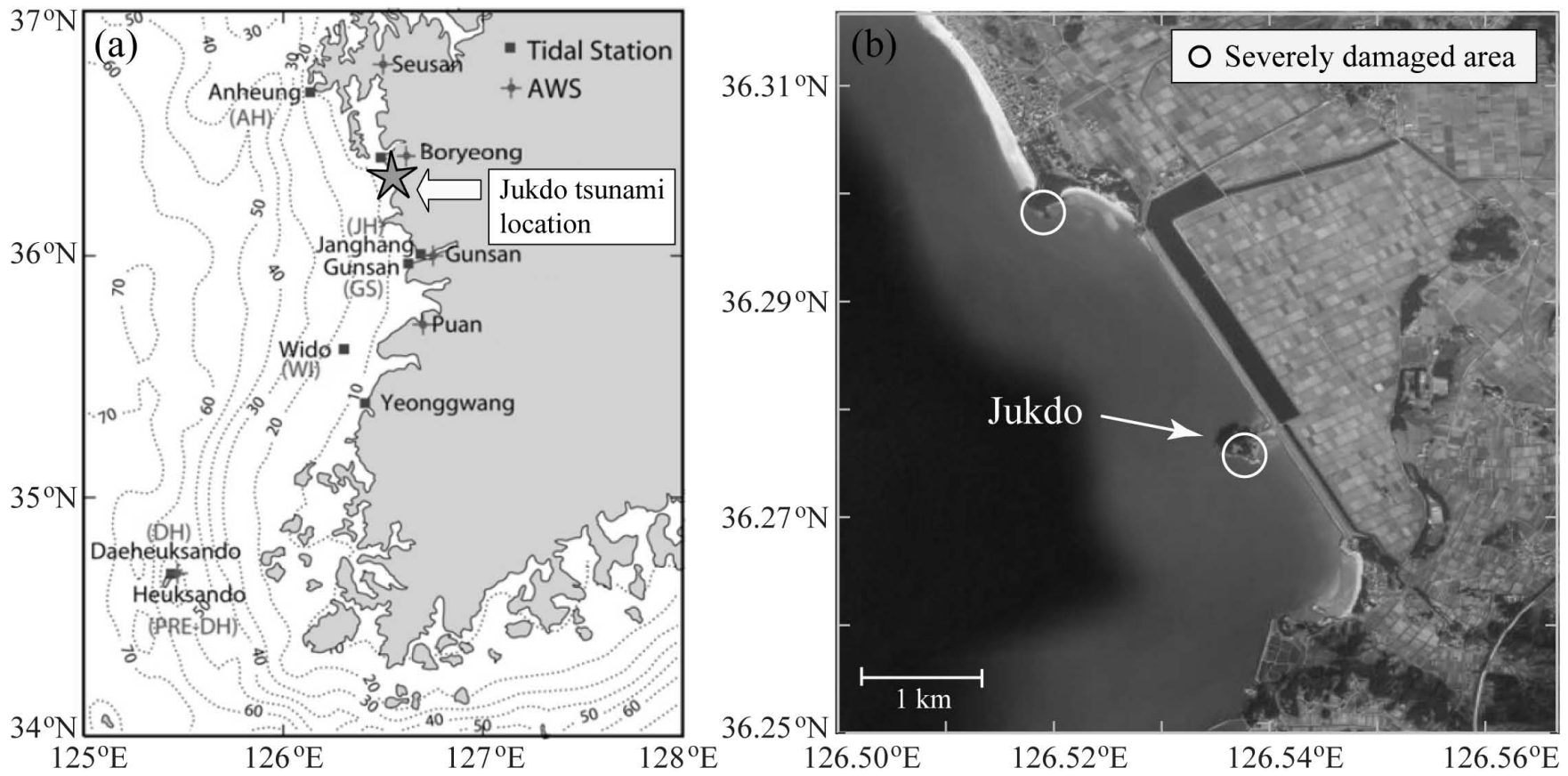

Fig. 1. (a) Location of the abnormal large and long waves observed on the west coast of Korea (i.e. Jukdo coastal pocket beach) on 4 May 2008 with marks of Tidal Stations (square) and Automated Weather Station (AWS) (plus), (b) and with indication of severely damaged areas and losses of life.

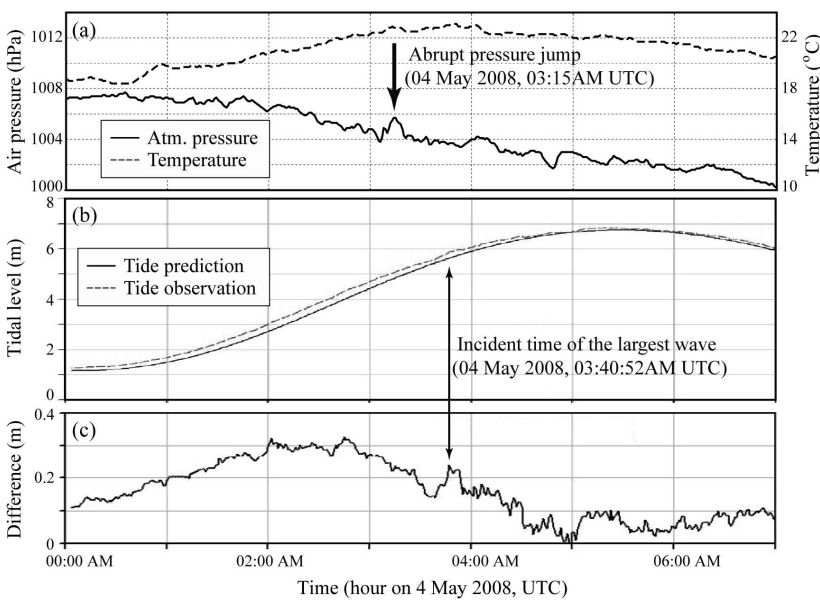

Fig. 2. (a) Variation of air pressure measured at the Boryeong AWS (Fig. 1) located close to Jukdo, (b) tidal level record and prediction at the Boryeong port located $15 \mathrm{~km}$ north of the Jukdo pocket beach, and (c) difference between the observed and predicted tide data.

However, a lack of reliable field measurement data is the key factor that limits a deeper understanding of meteorological contribution as one of the geo-mechanical sources to the generation of abnormal sea level oscillations. Even though a couple of tidal gauges were deployed near the pocket beach region, their records did not present any fully reasonable explanation of the disastrous tsunami-like wave event that took place at the entrance and inside the bay of the pocket beach (hereafter, referred to as Jukdo pocket beach or Jukdo bay). Fortunately, a CCTV surveillance system operated by a seafood restaurant at the entrance part of the pocket beach recorded the extreme sea level oscillations inside the Jukdo bay as well as the incoming open-ocean waves during the abnormal wave episode. Thus, it is expected that quantitative estimations of the tsunami-like sea surface oscillations from the CCTV video data will support a better understanding of the generation mechanism of the abnormal event initiated supposedly by the observed atmospheric disturbance.

This paper therefore will focus solely on estimating the characteristics of the tsunami-like abnormal waves using the video recordings through image calibration, processing and image analysis. Furthermore, frequency domain-based empirical tools such as stationary spectral and non-stationary wavelet analysis methods will be applied to the time-series of the video-derived sea-level signals to quantify the oscillations of the abnormal waves.

\section{Field surveys and video data}

Post-tsunami field surveys were conducted to collect physically meaningful landmarks and leftover tsunami-related features (Woo et al., 2008; among others), and to gain detailed descriptions of coastal damages and losses of human life around the Jukdo pocket beach (Oh et al., 2008). According to survey reports, severe damages and losses resulted in 

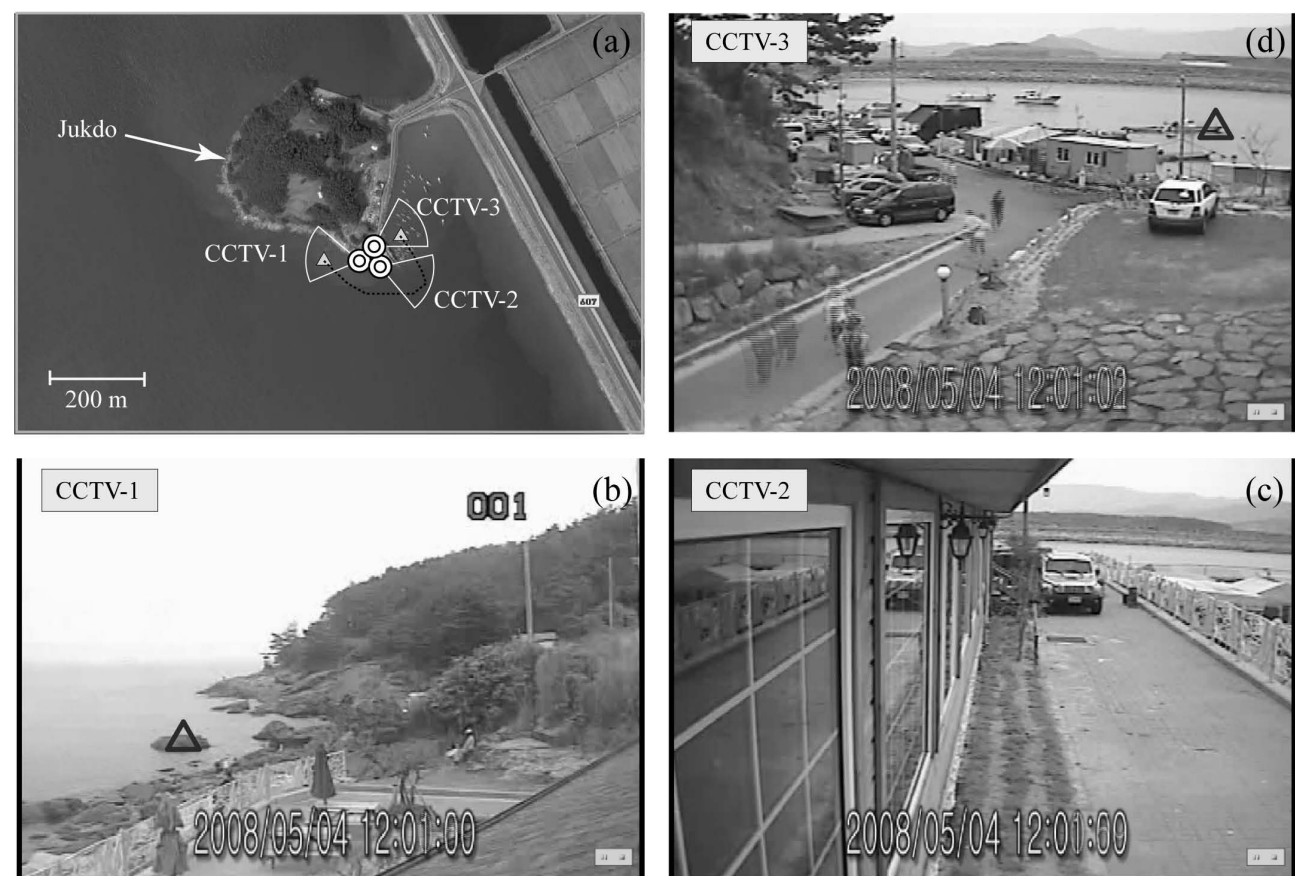

Fig. 3. (a) Location of a CCTV surveillance system deployed at the southern end of the Jukdo semi-island with three cameras facing different directions respectively. The dotted line is a path to measure a waterway distance for waves propagating between two different points (symbolized with triangles in b and d) defined in the first and third view sectors. (b), (c), and (d) Instantaneous snapshots from each video.

the two heads running out seaward as indicated with open circle marks in Fig. 1b. This coastal segment is separated into two sub-basins by the Jukdo semi-island in the middle of the coast. In total the two sub-basins have a width of approximately $5 \mathrm{~km}$ and a depth of approximately $4 \mathrm{~m}$ on average. It was stated by on-site eye-witnesses that the northern entrance part of each of the two sub-basins were severely inundated, resulting in catastrophic life losses of people fishing at the coastal line on the breakwater structures.

The field surveys could not find sound physical evidence for the tsunami-like wave heights which inundated the damaged area, due to the absence of reliable instrumental observations taken in the area. On the other hand, the tidal records sampled at every minute in the open-ocean and other nearby coastal locations (symbolized with closed squares in Fig. 1a) did not show the sea level oscillations amplified large enough to explain the vertical inundation scale around the Jukdo pocket beach. For instance, the Boryeong tidal station, located $15 \mathrm{~km}$ north of the Jukdo pocket beach, reported that the maximum height was about $0.3 \mathrm{~m}$ during the abnormal wave event (Fig. 2b and c).

However, the surveillance videos provided by the seafood restaurant clearly captured the tsunami-like strong oscillations as a physical eyewitness record, replaying the time sequence of the abnormal wave generation around the Jukdo pocket beach. Three CCTV cameras were positioned on the roof of the restaurant about $11 \mathrm{~m}$ above the Mean Water
Level (MWL), and about $15 \mathrm{~m}$ landward of the MWL contour. The three cameras collected video data at $1 \mathrm{~Hz}$ with sampling synchronization, obliquely observing the sea surface movements in three different directions (Fig. 3). The time in the CCTV system was found to run late two minutes and $45 \mathrm{~s}$, compared to local Korean standard time. The first camera, named CCTV-1, faced the incoming open-ocean from the entrance of the pocket beach at the southern end of the Jukdo semi-island, while the second and third cameras were directed toward the inner part of the bay.

Large parts of the video images captured by the first and third cameras cover the sea surface with fixed objects and features in the landward background, which are useful enough for the tsunami-like wave analysis. In contrast, the second video images contain only a small portion of the ocean surface in the rear part of the view, which makes it difficult to gain proper physical resolutions for the tsunami analysis. Therefore, only the video data collections from the first and third camera views were used to extract quantitative characteristics of the tsunami-like waves in this paper.

From each of the first and third video recordings, a video image time-series burst with a 59-min time-span was selected to include the abnormal sea-level oscillations of interest. Correspondingly, 3540-time-sequential images per burst having an 8-bit grayscale intensity depth were processed and analyzed. Table 1 summarizes the two video image bursts selected. Prior to the physical estimation of the oscillations 

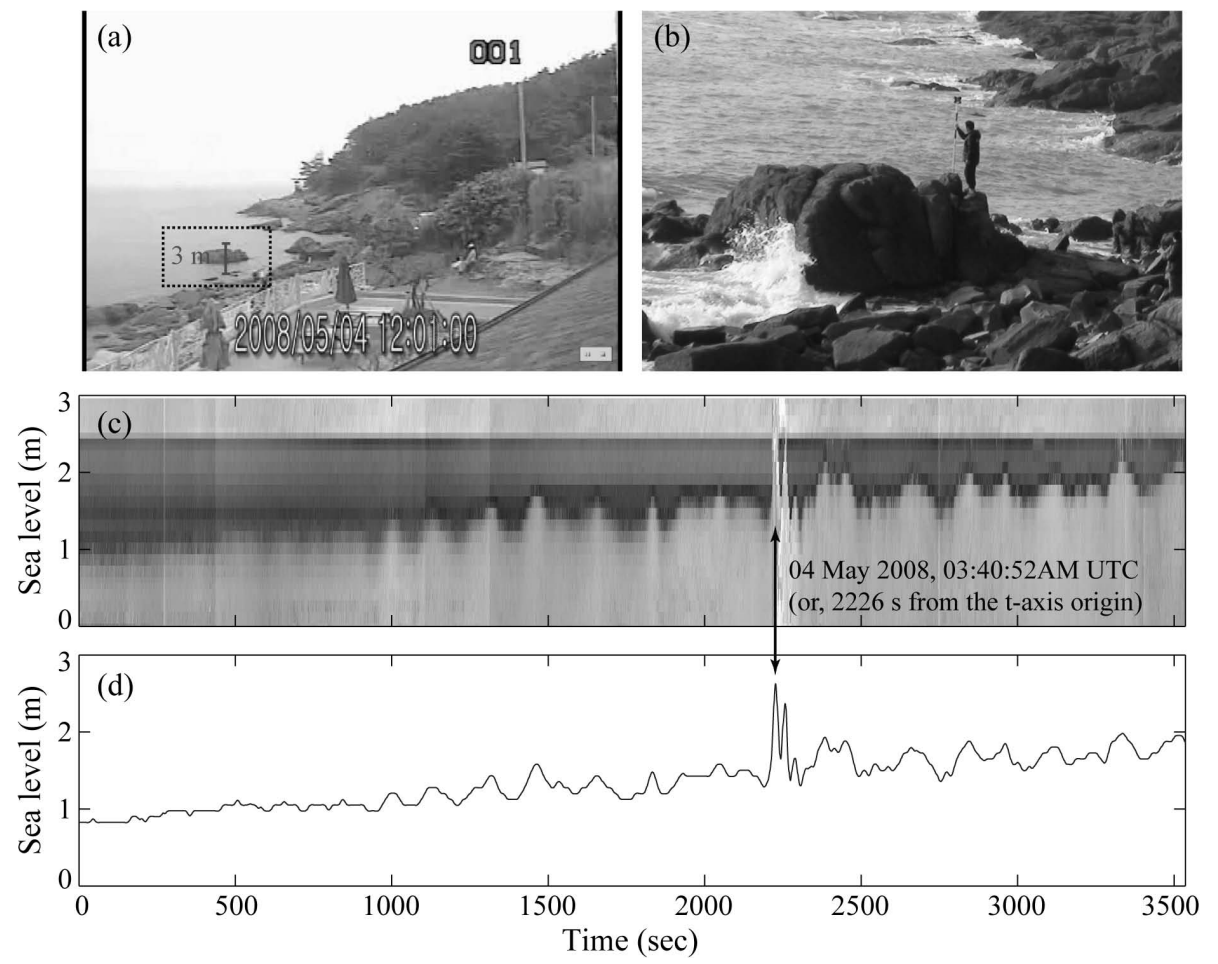

Fig. 4. (a) A rock column (shown in dotted rectangle) sticking out of the sea surface to quantify the vertical tsunami oscillations at the entrance of the Jukdo pocket beach, (b) scaling the lock height in image frame using the field measurement, (c) image timestack generated along the vertical $3 \mathrm{~m}$ high transect in (a) from the first video data burst, (d) time-series of sea-level variations extracted from the image timestack in (c) by means of the Canny edge detection method.

Table 1. Summary of the two video data bursts used in this paper.

\begin{tabular}{|c|c|c|}
\hline Parameters & CCTV-1 & CCTV-3 \\
\hline Date (UTC) & \multicolumn{2}{|c|}{4 May 2008} \\
\hline Start time (UTC) & \multicolumn{2}{|c|}{ 03:03:46 (hh:mm:ss) a.m. } \\
\hline Duration (min) & \multicolumn{2}{|c|}{59} \\
\hline Sampling rate $(\mathrm{Hz})$ & \multicolumn{2}{|r|}{1} \\
\hline Sector of view & $\begin{array}{l}\text { Seaside of the } \\
\text { pocket beach }\end{array}$ & $\begin{array}{l}\text { Inner side of the } \\
\text { pocket beach bay }\end{array}$ \\
\hline
\end{tabular}

from the video data, the calibration of the view sector of interest in the image frame was of critical importance to quantitatively extract the tsunami-like wave-related sequential information.

The photogrammetric transformation from image frame to real world coordinates requires the input of both the 2-D image coordinates and the 3-D world coordinates of visually discernable ground control points. A post-survey team from the Korea Ocean Research and Development Institute revisited the location of the video recordings to measure the ground control points from suitable fixed objects in the front parts of the two view sectors using a total station system.
Herein, the transformation from the video frame coordinates to real world coordinates was conducted using a direct linear transformation method (Holland et al., 1997). The accordingly rectified video images were re-sampled to have a regular pixel matrix and a pixel resolution of $0.1 \mathrm{~m} /$ pixel avoiding artifacts introduced during the image rectification process.

\section{Extraction of tsunami-related wave information}

\subsection{Coastal pocket-mouth sea level time-series}

Information of the vertical sea level oscillations can be obtained from typical monoscopic field video data, when a known physical scale is given to stick out of the oscillating sea surface within the video image frame (Suhayda and Pettigrew, 1977). Fortunately, the CCTV-1 video image frame contains a column-shaped steady rock sticking out of the vertically oscillating ocean, as shown in Fig. 4a. Thus, the vertical size of this rock was used to determine the physical scale of the sea level oscillations captured in the image domain.

The real height of the rock was measured during the revisit field survey of the ground control points (Fig. 4b). Thereby a vertically sticking $3 \mathrm{~m}$ high transect along the rock length could be defined with real length scale in the image frame corrected for the camera lens distortion in order to observe 
sea-level oscillations (Fig. 4a). The consequent physical resolution per vertical pixel turned out to be $0.075 \mathrm{~m} / \mathrm{pixel}$.

A 1-D image intensity array corresponding to the vertical transect was collected sequentially image-by-image from the CCTV-1 video data burst, yielding an image timestack which was consequently characterized by a 2-D physical plane $(t$, $z$ ), where $t$ and $z$ are the time and vertical coordinates respectively (Fig. 4c). The image timestack shows the apparent signature of the sea surface oscillations over the event time-span of interest. The trajectory of the vertical oscillations in time-series was identified by means of the Canny edge detection method (Canny, 1986), which was applied by running a $21 \times 21$ Gaussian filter (Height $\times$ Width in pixel) in the image timestack $(40 \times 3540$ pixels in the corresponding pixel scale). The trajectory identification by the edge detection method was empirically defined with two thresholds, i.e. 0.04 and 0.1 , typically required to consistently form continuous strong edges using image intensity gradients computed by the Gaussian filter. The resulting edge curve is presented in Fig. 4d, which will be used to determine the heights and periods of the tsunami-like oscillations.

\subsection{Inside-bay oscillation time-series}

Unlike the first video case, any appropriate solution for extracting the vertical sea-level oscillations provoked by the abnormal waves could not be found from the other video data burst captured in the direction toward the inside of the bay. Instead, the CCTV-3 video recorded free boats oscillating horizontally on the sea surface. The boats were floating at anchor, and bounced back and forth in the bay within anchor-chain bounds. The oscillations of the boats are therefore somewhat limited in their representation of the horizontal full length of the sea surface oscillations caused by the tsunami-like waves. For this reason, the horizontally oscillating motions of the boats are used here to indicate the timing of sea-level rise and fall in the bay.

Similar to the first video case, the extraction of the inner oscillation time-series involves defining a pixel array transect in real scale to generate an image timestack by collecting image intensity time-series along the transect from the video data burst, and identifying the trajectory of the horizontal oscillations of a boat in the image timestack. In this case, a $10 \mathrm{~m}$ long line transect for collecting time-series of a 1-D pixel intensity array is defined in the direction of the coastal pocket channel in the 2-D horizontal plane rectified with physical scale (Fig. 5a and b). The along-pocketchannel transect defined on the horizontal sea surface is used to trace bouncing motions of a boat (Fig. 5b).

Figure $5 \mathrm{c}$ shows the image timestack generated by collecting time-series of the 1-D image intensity array corresponding to the along-pocket-channel transect from the CCTV-3 video data burst. This image timestack shows the apparent continuous trace of the boat bouncing with the horizontal sea surface oscillations during the episode of interest. The tra- jectory of the horizontal oscillations in time-series was also extracted by means of the Canny edge detection method, using the same sub-window size and input parameter values. The resulting edge curve is presented in Fig. 5d, which will be used to gain information on the timing of either landward or seaward oscillation of the tsunami-like waves.

\subsection{Data analysis}

From the extracted signal record of the water surface oscillations along the vertical transect, tsunami height and rough period scale are investigated using a zero crossing-based time domain analysis. Frequency domain-based spectral analysis is also applied to examine the most dominant frequencies of the tsunami-like waves. Spectral analysis is carried out for the sea-level record of the total 3540 data points by using a moving interrogation window size of 2048 points with $50 \%$ overlapping. The resulting spectral estimates have a frequency resolution of $0.000488 \mathrm{~Hz}$ and 3.9 degrees of freedom (Nuttall, 1971). The corresponding lower and upper multipliers for the $95 \%$ confidence intervals on spectral peaks are 0.4 and 6.0, respectively (Jenkins and Watts, 1986).

In addition, a wavelet analysis is applied to the nonstationary oscillation signal to obtain transient wave period which is defined as a wave period corresponding to the instantaneous peak energy in the wavelet spectrum at each time point. The wavelet analysis is carried out by using the continuous Morlet wavelet transform (Morlet et al., 1982) which is useful for time-series analysis and for capturing oscillatory behavior (Torrence and Compo, 1998).

Besides the frequency analysis of the abnormal waves, actual speed of the tsunami propagations is estimated by computing the wave travel time between the point-measurement pairs provided from the two video data bursts, since synchronization of the video recordings was available during the abnormal wave event. Thus, phase speeds of the tsunami waves are computed by:

$c=\frac{\Delta s}{\Delta t}$

where $c$ is phase speeds of waves, $\Delta s$ is a distance between two different locations, and $\Delta t$ is the travel time over the distance $\Delta s$. Theoretically, phase speed of extremely long waves can be derived from shallow-water wave theories (e.g. linear shallow-water wave celerity is given by the expression $c=\sqrt{g h}$, where $g$ is the gravitational acceleration and $h$ is the mean water depth). However, since the celerity of large and long shallow-water waves is influenced by both the wave height $H$ and the water depth $h$, the below formula of the solitary wave celerity theory is used here for a comparison with the celerity estimates:

$c=\sqrt{g(h+H)}$. 

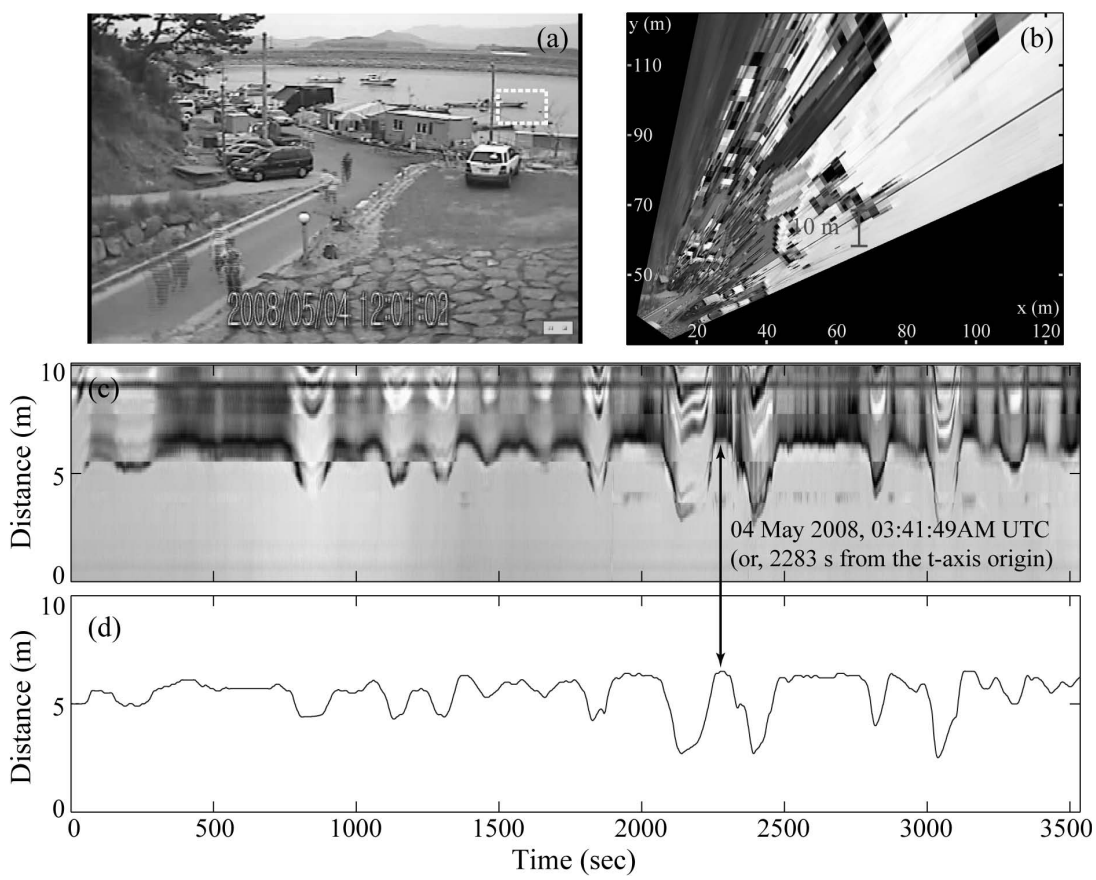

Fig. 5. (a) A boat used to measure horizontal sea-level oscillations in the third video view sector, (b) a image pixel array to track the horizontal movements of the boat in (a) in time sequence, (c) image timestack generated along the 10m-long transect in (b) from the third video data burst, (d) time-series of horizontal sea-level oscillations extracted from the image timestack in (c) by means of the Canny edge detection method.

\section{Results and discussion}

This paper investigated only a time-interval spanning about one hour and containing the strongly amplified sea-level oscillations, that were noticeably observed in the view sectors of the video images. The analysis of the video recordings was therefore focused on the characteristics of the tsunamilike oscillations analyzable within this time frame. Mainly, heights, periods, and propagation speeds of the oscillations were quantitatively extracted from the two video data bursts recorded in the two different directions of the incoming openocean waves and the inside part of the bay.

\subsection{Tsunami height and period}

In order to carry out the analysis of the tsunami-like waves unexpectedly occurred at the local Jukdo pocket beach, the vertical sea-level oscillation signal derived from the first video burst was detrended by removing the best straight-line fit to begin with, as shown in Fig. 6a. It is clearly seen that the detrended time-series were not stationary, due to the occasional tsunami-like wave incidence to the pocket beach region.

A time domain-based analysis of the detrended data using the zero down-crossing method resulted in a trend that the trough-to-crest height of the sea-level oscillations with wave periods ranging from 26 to $232 \mathrm{~s}$ gradually increased and slowly decayed after the happening of the maximum coastal inundation at $2226 \mathrm{~s}$ with regard to the start time of the video burst (Fig. 6a). The consequent average of the abnormal wave heights is $0.3 \mathrm{~m}$. Interestingly, the largest trough-tocrest oscillation about $1.3 \mathrm{~m}$ high appeared at the entrance of the pocket beach region after the unusual amplification increase of the oscillations lasted tens of minutes. The largest sea-level rise from the trough to the crest was almost immediate, within $36 \mathrm{~s}$, compared to other oscillation intervals.

The spectral analysis of the detrended time-series provides more resolvable frequency and energy information of the tsunami-like oscillations, as presented in Fig. 6b. The variance density spectrum in the long wave frequency band (i.e. less than $0.05 \mathrm{~Hz}$ ) shows a decay trend with regard to the increase of frequency $f$ (i.e. expressed in a form of $f^{-\alpha}$ where $\alpha$ is a typically positive constant empirically determined), as found in earlier seiche-related studies (Veraart, 1994; de Jong and Battjes, 2004). In line with the decay trend, three main energy peaks are found at $0.00098,0.0054$, and $0.031 \mathrm{~Hz}$ of which the first two are the most considerable. The significant height of the oscillations computed from the energy density spectrum using $4 \sqrt{m 0}$, where $m 0$ is zero moment of the spectrum, is about $0.6 \mathrm{~m}$. The wind-driven gravity waves did not appreciably contribute to the zero-moment-based height of the tsunami-like long waves, as found in the energy spectrum. 
Since the sea-level oscillations are not homogeneous in time, the three energy peaks distributed in the frequency domain itself do not allow to describe how the oscillation frequencies changed during the tsunami-like wave episode. Instead, the wavelet transform analysis of the time-series yielded two dimensional spectrum of wave energy in timefrequency domain (Fig. 6c), showing dominant frequency variability with regard to time. The spectrum was normalized with the maximum energy value within the domain. From Fig. 6c, time-localized three major energy peaks are found. The first and third energy peaks having a similar wave period band ranging 160 to $210 \mathrm{~s}$ are found between 1000 and $1800 \mathrm{~s}$, and between 2050 and $3000 \mathrm{~s}$ in the time frame, respectively. At the time of the largest sea-level rise observed within the time-window of the third energy peak, another shorter wave period less than $1 \mathrm{~min}$ is also outstanding. In contrast, the second peak with a much longer wave period band ranging from 850 to $1050 \mathrm{~s}$ strongly appears between the time-spans of the first and third peaks. The transient peak wave frequency bands from the wavelet analysis are consistent with the three dominant frequencies obtained through the spectral analysis, and even further quantitatively reveal an evolution pattern of the abnormal ocean oscillations in time.

\subsection{Propagation direction and speed}

Direction and phase speeds of the tsunami-like waves could be estimated from the rectified image sequence of the first video data burst. Time-sequential image snapshots captured during the largest wave-crest appearance in the view sector of the first video are presented in Fig. 7. The largest wave surface elevation was characterized by a wave breaking-induced foam, which resulted in non-discrete image patches. Consequently, it can be seen from the non-discrete image patterns that the largest wave crest propagated along the shore around the southern half of the Jukdo semi-island in a counterclockwise direction. In addition, Fig. 7c shows that the largest wave crest characterized by a bright long line band was incoming from NNW.

The three successive images selected are temporally spaced with a time interval $d t=3 \mathrm{~s}$, to reduce ambiguity of the non-discrete image pattern flows. The displacements of the largest wave crest breaker $(d s)$ per time interval are approximately $22 \mathrm{~m}$ on average, estimated by manual tracking of the non-discrete image patterns. Therefore, the phase speed of the largest wave incoming in the entrance part of the Jukdo pocket beach was computed as $c(=d s / d t)=7.3 \mathrm{~m} / \mathrm{s}$ using Eq. (1).

In addition, the overall phase speed of the abnormal waves was calculated by computing the travel time over a long distance between the two different locations selected from the first and third video view sectors. The first one is the stone column sticking out of the water defined within the first video sector, and the other one is the boat horizontally oscillating with the ocean in the third video. Figure 8 provides a com-
Table 2. Tsunami propagation speeds computed by computing the travel time over a distance between two different locations selected from the first and third video view sectors. A and A', B and B', C and C', and D and D' are time point pairs representing the travel times of individual tsunami waves (Fig. 8).

\begin{tabular}{lcccc}
\hline Time point & $\begin{array}{c}\mathrm{A} \\
(998 \mathrm{~s})\end{array}$ & $\begin{array}{c}\mathrm{B} \\
(1318 \mathrm{~s})\end{array}$ & $\begin{array}{c}\mathrm{C} \\
(1463 \mathrm{~s})\end{array}$ & $\begin{array}{c}\mathrm{D} \\
(2226 \mathrm{~s})\end{array}$ \\
\hline $\begin{array}{l}\text { Time-lag } d t(\mathrm{~s}) \\
\text { Distance } d s(\mathrm{~m})\end{array}$ & $63\left(\mathrm{~A}^{\prime}-\mathrm{A}\right)$ & $54\left(\mathrm{~B}^{\prime}-\mathrm{B}\right)$ & $65\left(\mathrm{C}^{\prime}-\mathrm{C}\right)$ & $57\left(\mathrm{D}^{\prime}-\mathrm{D}\right)$ \\
Phase speed $(\mathrm{m} / \mathrm{s})$ & 6.1 & 7.1 & 582 & 6.7 \\
\hline
\end{tabular}

parison of the two wave signals extracted at the two locations, showing time lags of four selected individual wave crests propagating between them. Table 2 summarizes their overall phase speeds computed by using Eq. (1). The distance between the two locations was measured along a possible wave path determined based on the bottom contours from a high resolution topographical map, as shown in Fig. 3c. The overall phase speed ranges from 5.9 to $7.1 \mathrm{~m} / \mathrm{s}$. Considering the decrease of water depth by about $1 \mathrm{~m}$ along the path from the entrance to the inner part of the pocket beach area, the overall speed range is consistent with the phase speed of $7.3 \mathrm{~m} / \mathrm{s}$ that was computed within the view sector of the first video.

Based on the celerity of solitary wave theory (Eq. 2), the inversely computed water depth $h$ and tsunami height $H$ were compared to the directly measured height and depth. The phase speed $7.3 \mathrm{~m} / \mathrm{s}$ in the entrance part of the bay is inverted to $h+H=5.4 \mathrm{~m}$ using Eq. (2). Since the water depth at the entrance is about $4 \mathrm{~m}$ on average, the tsunami height is deduced to be $1.4 \mathrm{~m}$ which is comparable to the measured height of $1.3 \mathrm{~m}$. The estimated overall phase speeds also yields an inverted depth plus height range of 3.5 to $5.1 \mathrm{~m}$, which is consistent with the decrease of the depth along the path of wave propagation.

Many losses of life were caused by the largest trough-tocrest height of the tsunami-like oscillations in the entrance part of the basin, in which the height and propagation speed were found to be about $1.3 \mathrm{~m}$ and $7.3 \mathrm{~m} / \mathrm{s}$, respectively. The abrupt sea-level rise from the temporal mean sea level to the largest crest occurred within about $18 \mathrm{~s}$, computed from the sea-level time-series in Fig. 6a. These facts illustrate that people focusing on fishing on a nearby shore could not survive the disastrous waves unless they escaped immediately at a pace much faster than the wave speed of $7.3 \mathrm{~m} / \mathrm{s}$ within such a short term interval.

\subsection{Sources of measurement errors}

The tsunami-like ocean surface oscillations were quantified from the time-series of the CCTV images physically calibrated using the geometric field survey data. Possible error sources in measuring the abnormal large waves are: 1) errors in the field survey, and 2) errors in calibrating the image frame into the real physical scales. 


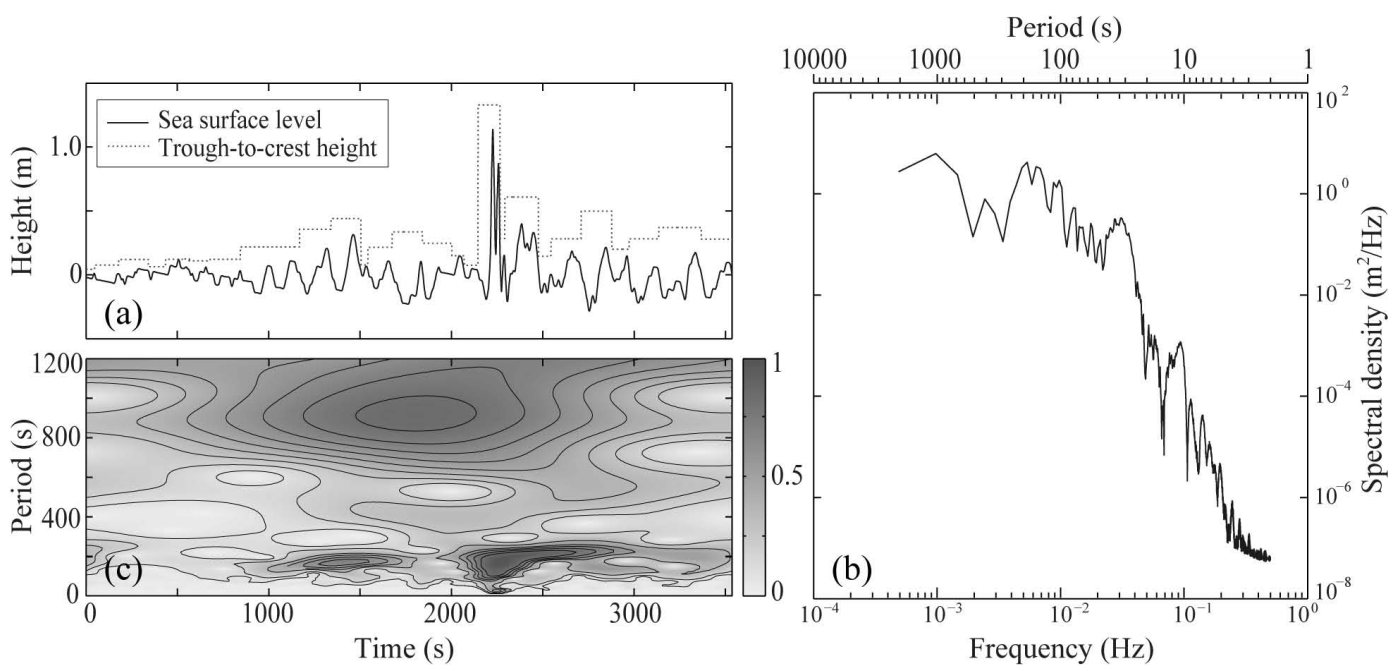

Fig. 6. (a) the detrended time-series of the vertical sea-level oscillations in Fig. $4 \mathrm{~d}$ and trough-to-crest height of the tsunami-like waves computed through the zero up-crossing method, (b) energy spectrum of the detrended time-series in (a), (c) wavelet energy spectrum normalized by the maximum energy value.
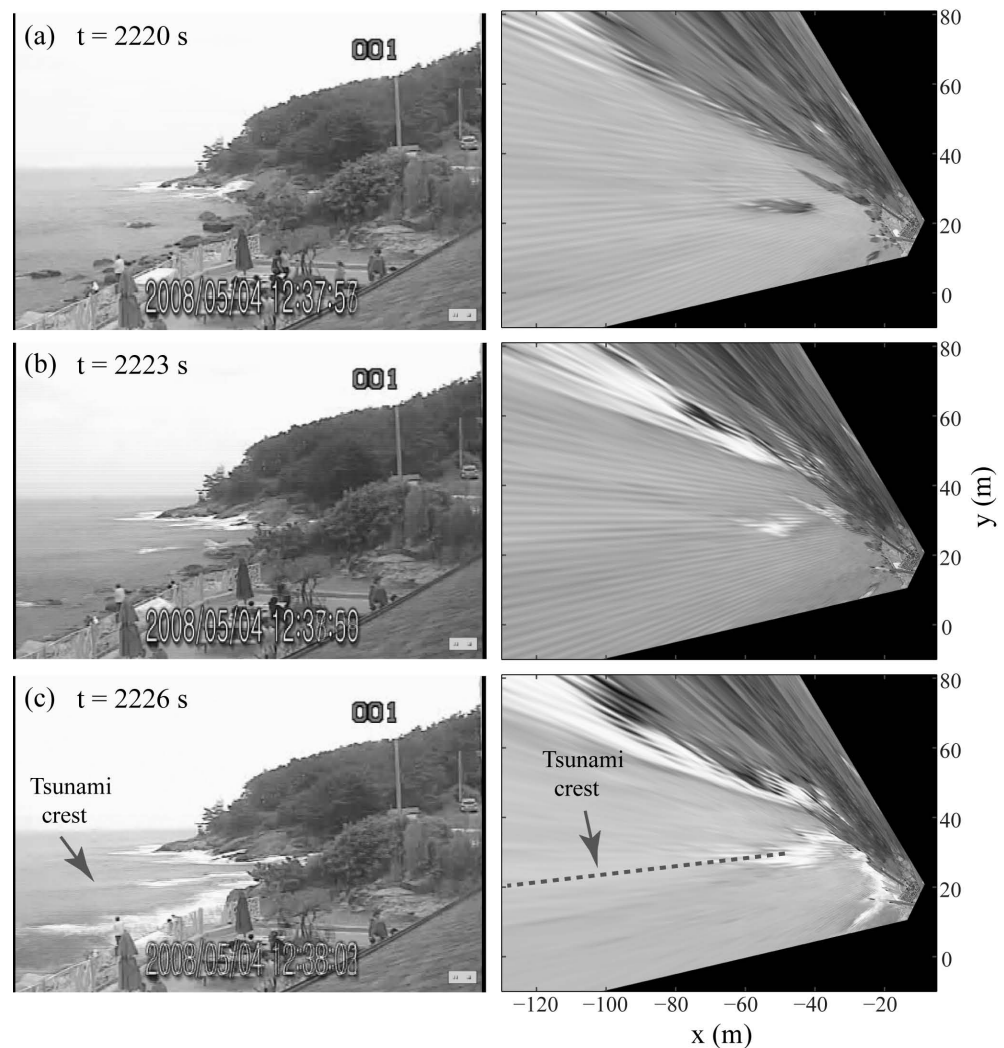

Fig. 7. Sequential images of the largest abnormal wave propagating the coastal pocket mouth area during the tsunami episode. (Left panel) three successive images with a time interval of $3 \mathrm{~s}$, and (right panel) their rectified images.

Errors in surveying the field geometry might be caused by human handling of the total station system and accuracy of the instrument as well. However, the human error source is to be considered minimal as much as the round-off error of the measuring scale by the instrument, since the survey scientists were educated and trained well enough to survey the field geometry within the error range the manufacturer documented. The round-off errors by the rod and station system were up 

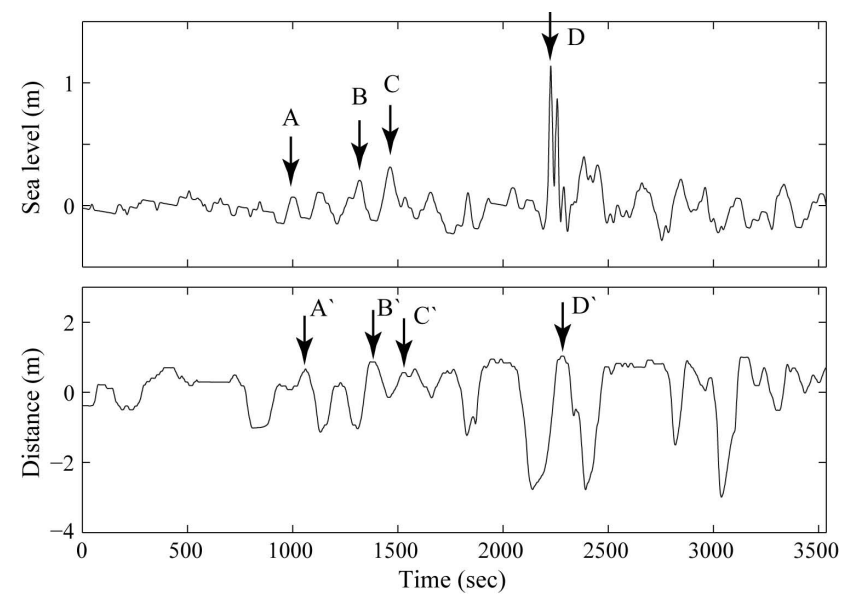

Fig. 8. Comparison of the two sea-level oscillation time-series extracted from the first and third video data bursts, respectively. A and A', B and B', C and C', and D and D' are time point pairs showing the time-lags of individual tsunami waves travelling between the two video view sectors.

to $0.005 \mathrm{~m}$ in the vertical over the surveyed distance of about $100 \mathrm{~m}$, thereby being negligible compared to the measured sea surface oscillations that are in the order of $1 \mathrm{~m}$.

One of the image calibration processes involved here was to physically scale the sea surface oscillations along a 1-D pixel array in the image frame corresponding to the vertical transect defined along the rock column sticking out of the ocean surface. The 1-D pixel array (40 pixel long and free from lens distortion) was linearly matched with the vertical transect of $3 \mathrm{~m}$, resulting in the physical resolution per pixel being equal to $0.075 \mathrm{~m} /$ pixel. During this image calibration, manual selections of two different identifiable image pixels (i.e. the top of the rock and the bottom of the rock) along the vertical transect were required to register the collected pixels as the physical references. Repeated tests for the manual selections in the image frame revealed that the maximum deviation from the average of the selected pixels was two pixels. Two-pixel deviations at each of the two reference points, in the worst case, can introduce a measurement error up to about $10 \%$ systematically by this image calibration.

Another image calibration process was to transform the 2D image coordinates to real-world coordinates. Physical distance errors in the image rectification process were evaluated using the surveyed ground points and the predicted locations of them in the rectified image frame, as presented in Table 3. The physical distance errors computed for the CCTV-1 and CCTV-3 cases were about 0.16 and $0.10 \mathrm{~m}$, respectively, on average. Since the rectified images were used to measure the horizontal sea surface motions in the order of $10 \mathrm{~m}$, the physical distance errors by the image rectification process are negligible.
Table 3. Image rectification errors estimated using the surveyed ground points and the predicted locations of the ground points in the rectified domain. The physical distance errors at individual points were averaged.

\begin{tabular}{lcc}
\hline Case & \multicolumn{2}{l}{ Physical distance error $(\Delta l)$} \\
\cline { 2 - 3 } & $\begin{array}{l}\text { \# of surveyed } \\
\text { ground points }\end{array}$ & $\Delta l$ \\
\hline CCTV-1 & 11 & $0.16 \mathrm{~m}$ \\
CCTV-2 & 19 & $0.10 \mathrm{~m}$ \\
\hline
\end{tabular}

The investigations above revealed that the error sources during the field surveys and the image rectification process are negligible, while the error in physical scaling of the 1-D image array to measure the vertical sea surface oscillations can cause measurement errors up to $10 \%$. This fact suggests that accumulated measurement errors can be at most $10 \%$.

\section{Conclusions}

In order to investigate the characteristics of a tsunami-like wave event that occurred in a local coastal pocket beach of the west coast of Korea on 4 May 2008, CCTV videos that captured the episode were processed and analyzed in this paper. Quantitative information of the vertical and horizontal sea surface oscillations were extracted from two video data bursts sampled at $1 \mathrm{~Hz}$ with a time-span of about one hour: one is for estimating vertical oscillating wave motions at the entrance of the basin, and the other is for tracking the trajectory of the waves with time in the inner part of the coastal pocket region. The quantification processes from the two recordings involved calibration of image distortions, accumulation of the time-series of a 1-D intensity array along a transect of interest defined in the calibrated image view sector, and identification of the trajectory of the oscillations revealed in time along the transect. The analysis of the extracted wave signals by means of time and frequency domain-based statistical methods show that the maximum trough-to-crest height of the abnormal waves reached $1.3 \mathrm{~m}$, having a dominant period of $185 \mathrm{~s}$ (3.1 min). In addition, computation of wave phase speeds by counting time-lag of propagation over a known distance revealed that the largest wave speed was about $7.3 \mathrm{~m} / \mathrm{s}$, which caused the deaths of many victims nearby the shore who could not escape immediately faster than the quick tsunami flooding speed.

Acknowledgements. This work was supported by the Korea Ocean Research \& Development Institute (Grant PE98452 and PE98453). This research was also supported in part by a grant from "Development of Korea Operational Oceanographic System (KOOS)" 
funded by the Ministry of Land, Transport and Maritime Affairs of the Korean government. The authors wish to thank Gye-Jong Lee, the owner of the Bomulseom restaurant at the Jukdo, for supplying the CCTV video data, and Hak-Yoel You at KHOA for providing technical assistance with the use of the video data.

Edited by: S. Tinti

Reviewed by: G. Iglesias and another anonymous referee

\section{References}

Canny, J.: A computational approach to edge detection, IEEE T. Pattern Anal., PAMI-8(6), 679-698, 1986.

Choi, B.-J., Park, Y., and Kwon, K.: Generation and growth of long ocean waves along the west coast of Korea in March 2007, Ocean and Polar Research, 30(4), 453-466, 2008 (in Korean).

De Jong, M. P. C. and Battjes, J. A.: Low-frequency sea waves generated by atmospheric convection cells, J. Geophys. Res., 109(C1), C01011, doi:10.1029/2003JC001931, 2004.

Dragani, W. C., Mazio, C. A., and Nunez, M. N.: Sea level oscillations in coastal waters of the Buenos Aires province, Argentina, Cont. Shelf Res., 22, 779-790, 2002.

Goring, D. G.: Rissaga (long-wave events) on New Zealand's eastern seaboard: A hazard for navigation, in: Proc. 17th Australasian Coastal Ocean Eng. Conf., 20-23 September 2005, Adelaide, Australia, 137-141, 2005.

Hibiya, T. and Kajiura, K.: Origin of "Abiki" phenomenon (a kind of seiches) in Nagasaki Bay, Journal of Oceanographic Society of Japan, 38, 172-182, 1982.

Holland, K. T., Holman, R. A., and Lippmann, T. C.: Practical use of video imagery in near-shore oceanographic field studies, IEEE J. Oceanic Eng., 22(1), 81-92, 1997.

Jenkins, G. M. and Watts, D. G.: Spectral analysis and its applications, Holden-Day, San Fransisco, CA, USA, 525 pp., 1986.

Monserrat, S. and Thorpe, A. J.: Gravity wave observations using an array of microbarographs in the Balearic Islands, Q. J. Roy. Meteor. Soc., 118, 259-282, 1992.

Monserrat, S., Vilibić, I., and Rabinovich, A. B.: Meteotsunamis: atmospherically induced destructive ocean waves in the tsunami frequency band, Nat. Hazards Earth Syst. Sci., 6, 1035-1051, 2006, http://www.nat-hazards-earth-syst-sci.net/6/1035/2006/.
Morlet, J., Arens, G., Fourgeau, E., and Giard, D.: Wave propagation and sampling theory: Part I. Complex signal and scattering in multilayered media, and Part II. Sampling theory and complex waves, Geophysics, 47, 203-221, 222-236, 1982.

Nuttall, A. H.: Spectral estimation by means of overlapped FFT processing of windowed data, Naval Underwater Systems Center Report No. 4169, New London, CT, USA, 1971.

Oh, Y.-M., Kang, T.-S., Jung, K.-H., Um, K.-S., and Hong, S.-J.: Final report of field survey to coastal damages by abnormal waves around the Boryeong Jukdo, Technical Report by the Coastal Disaster Prevention Council, National Emergency Management Agency, Seoul, Korea, 2008 (in Korean).

Papadopoulos, G. A.: Some exceptional seismic (?) sea-waves in the Greek Archipelago, Science of Tsunami Hazards, 11, 25-34, 1993.

Rabinovich, A. B.: Long ocean gravity waves: trapping, resonance and leaking, Gidrometeoizdat, St. Petersburg, 1993 (in Russian).

Suhayda, J. N. and Pettigrew, N. R.: Observations of wave height and wave celerity in the surf zone, J. Geophys. Res., 82(9), 1419$1424,1977$.

Torrence, C. and Compo, G. P.: A practical guide to wavelet analysis, B. Am. Meteorol. Soc., 79(1), 61-78, 1998.

Veraart, C.: Events of long waves in the North Sea, Tech. Rep. RIKZ-94.034, Rijkswaterstaat/RIKZ and Delft University of Technology, Delft, The Netherlands, 1994.

Vilibić, I. and Beg Paklar, G.: High-frequency atmosphericallyinduced oscillations in the middle Adriatic coastal area, Ann. Geophys., 24, 2759-2771, 2006, http://www.ann-geophys.net/24/2759/2006/.

Wang, X., Li, K., Yu, Z., and Wu, J.: Statistical characteristics of seiches in Longkou Harbour, J. Phys. Oceanogr. 17, 1063-1065, 1987.

Woo, S. B., Seung, Y. H., Yoo, H. R., and Oh, Y. R.: Investigation of possible sources for generation of abnormal waves in May 2008 using wavelet analysis, in: Workshop on 2008 Boryeong Abnormal Waves, Korean Society of Coastal and Ocean Engineers, Jeju, Korea, 29 May 2008. 